\title{
Combination of polymer sensors with organic electronics: ideas and developments
}

\author{
G. Domann, U. Helbig, \\ Fraunhofer-Institut für Silicatforschung ISC, Neunerplatz 2, 97204 Würzburg (Germany); \\ M. Zirkl, B. Stadlober, \\ Joanneum Forschungsgesellschaft mbH, Franz-Pichler-Str. 30, 8160, Weiz (Austria)
}

\section{$\underline{\text { Introduction }}$}

Step by step, everyday items are more and more featured with sensor functions in order to increase the item's capability for self-controlling, self-monitoring or communication with other devices and people. Some of the most interesting physical properties which are to be measured by sensors are pressure and temperature (infrared radiation). In order to equip commodity items with sensor functions it is often necessary to apply efficient processing techniques in order to reduce the costs. Low production costs can be realized by large-area processing techniques, such as reel-to-reel technologies using printing and imprinting tools, and, in-line vacuum processing schemes, as well.

Since several years, research activities are carried out in order to realize printable electronics. The combination of printable sensors and printable electronics enables one to increase the degree of device integration within the same process line. Besides, experience gained in the field of research of printable electronics can be exploited in order to achieve printable pressure and temperature sensors. In a first approach, simple organic thin-film transistors are applied in order to match the impedance of the sensor devices and to amplify the sensor signal.

By the combination of printable electronics and printable sensors two further interesting features become available:

(1) The active sensor area can be increased in arbitrary sizes and shapes since it is dependent on the web width of the processed foil only and can be cut or formed in adjusted shapes defined by the final application.

(2) By usage of printable electronics it becomes possible to feature the sensor with position sensitivity Therefore, the complexity of information gathered by the sensor can be increased and enables one to realize more sophisticated functions without increasing the processing costs.

However, transforming the idea into reality many requirements have to be met by the used materials, the device physics, and the process technologies. These aspects are discussed in detail in this contribution and main research topics are identified.

\section{The sensor concept}

The sensor concept was described by Zirkl et al [1]. A pyroelectrical material film is embedded between electrodes. Due to a change of temperature a voltage signal is generated. This signal is used as gate voltage for a thin film transistor controlling the output current $I_{D S}$. This concept can be applied for several approaches: The sensor material might be a ferroelectric polymer featured by piezo- or pyroelectric properties. As well, electret foams can be used in order to realize a capacitive pressure element. There are also several possibilities to implement the transistor structure using organic semiconductors on basis of polymers, such as $\mathrm{P} 3 \mathrm{HT}$, or small molecules, for example pentacene. Besides, it is also feasible to integrate electrochemical transistors as described by Nilsson et al. [2].

A schematic picture of the sensor element in combination with a printed organic thin film transistor is displayed in Fig. 1. In this case, PET was used as substrate, a fluorinated ferroelectret polymer as sensor material, pentacene as organic semiconductor and a nanocomposite as gate dielectric. 


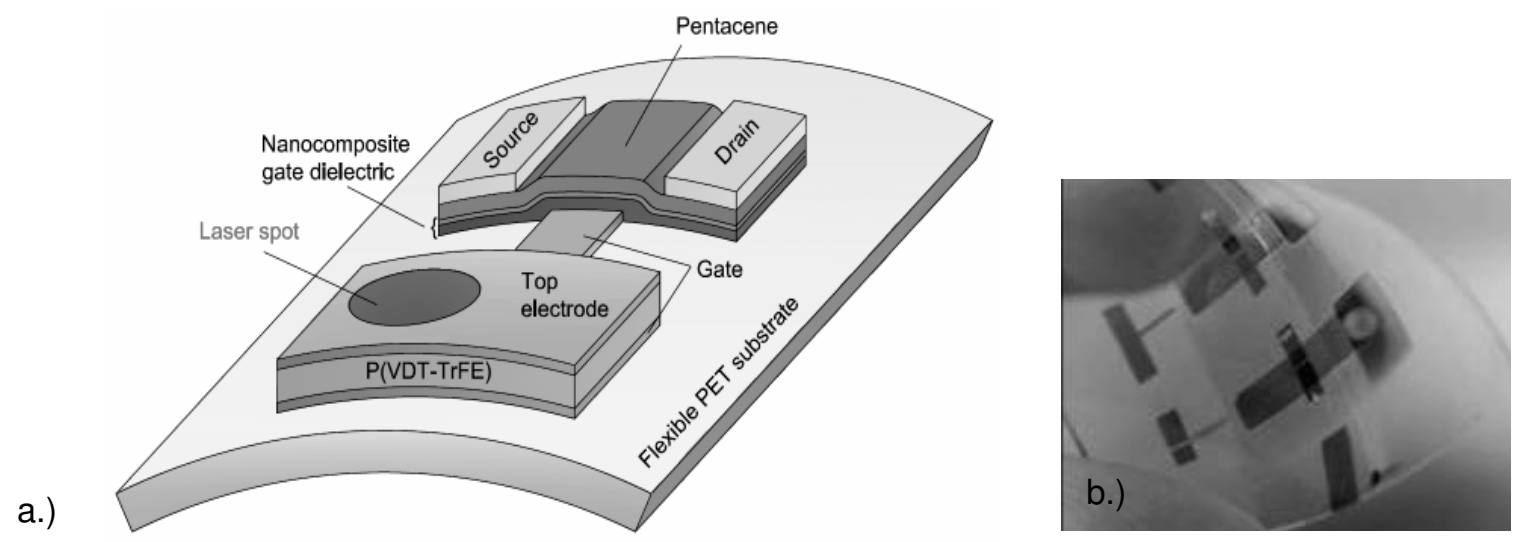

Figure 1: a.) A scheme of printable sensor elements using ferroelectric fluorinated polymers, pentacene as organic semiconductor, and nanocomposite as gate dielectric. Alternatively, inorganic-organic hybrid polymers (ORMOCER ${ }^{\circledR}$ s) can be used; b.) a realized lab-scale sensor device which is activated by a laser spot.

These sensor devices are sensitive against infrared radiation and exhibit a reproducible sensor signal, even after several hundreds of switching cycles. The output current may vary about five orders of magnitude in dependence on the sensor excitation. This performance enables one to use these kinds of sensors for a variety of application. In figure 2, the switching behavior of a sensor device is demonstrated by means of the produced output current. No degradation of the activity of the sensor material can be observed. Moreover, the sensor detects not only the existence of a radiation source but also the distance. Approaching a finger tip to the sensor, the output current varies in dependence on the distance. This signal generation of an approaching finger can be used as a touch-less keypad when the vertical distance is also detected by a second sensor device,

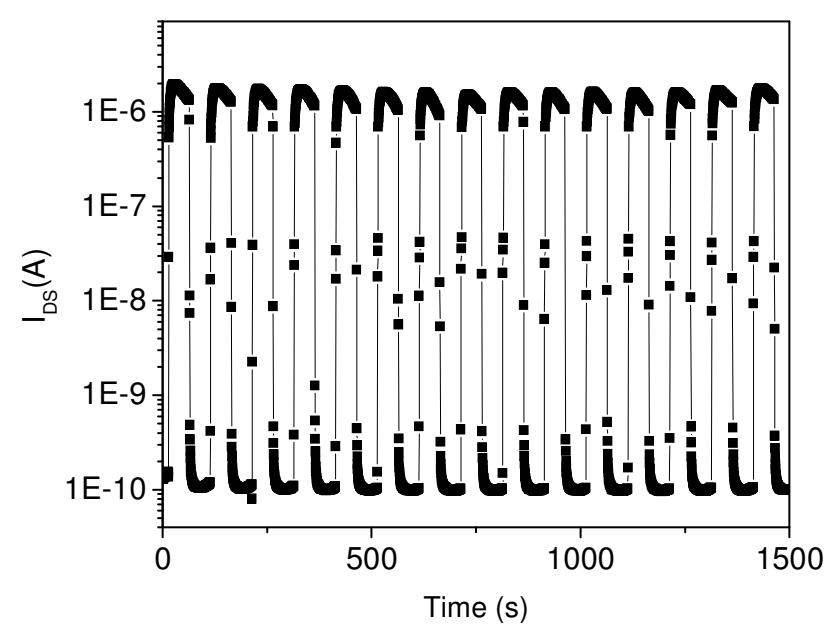

Figure 2: The output current of an organic TFT controlled by a polymer sensor device which is excited by a laser source.

\section{Pyroelectric polymers}

When starting the process development for large area sensor devices a metalized PET foil is used. The electrodes and conductor lines are patterned by means of reel-to-reel UV lithography or subtractive mechanical patterning. In a second step, the sensor material is applied by a screen-printing process. This sensor material is a ferroelectric fluorinated copolymer from the PVDF family. Such copolymers offer the advantage of direct crystallisation of the ferroelectric phase from solution without further treatment. 
Previous investigations showed that PVDF based pyroelectric sensor films of high quality can be prepared from liquid precursors [3]. The precursors were prepared diluting the copolymer in an appropriate solvent and subsequent spin coating and annealing.

For large area devices it is planned to apply the sensor film via screen printing. For that process specific rheological properties of the precursor are required.

The screen printing process can be divided in three phases: the filling phase, the contact phase and the release phase. During these phases the ink or paste is exposed to different shear forces. During the filling phase and the release phase low shear forces are applied whereas the contact phase is correlated with high shear forces. To achieve a good printing result concerning surface quality and resolution a certain thixotropic behaviour of the printing pastes or inks is required.

As the viscosity and the rheological behaviour of the precursor solutions developed for spin coating do not match the requirements for screen printing, the solutions have to be modified adjusting the solvent system and specific additives.

At first, polymer solutions were prepared based on several solvents. Thin films were prepared by bar coating and the films were investigated concerning homogeneity and phase content after annealing.

Due to the solubility of PVDF, $y$-butyrolactone (GBL), N-methyl pyrrolidone (NMP), and dimethyl sulfoxide (DMSO) were chosen as solvent.

The cured films were characterized using X-ray diffraction, ATR-FTIR spectroscopy and polarization microscopy.

The analysis of the phase content via X-ray diffraction and IR spectroscopy showed that all films consist of the intended $\beta$-phase of the PVDF copolymer (Fig.3). All films appear homogeneous after annealing.

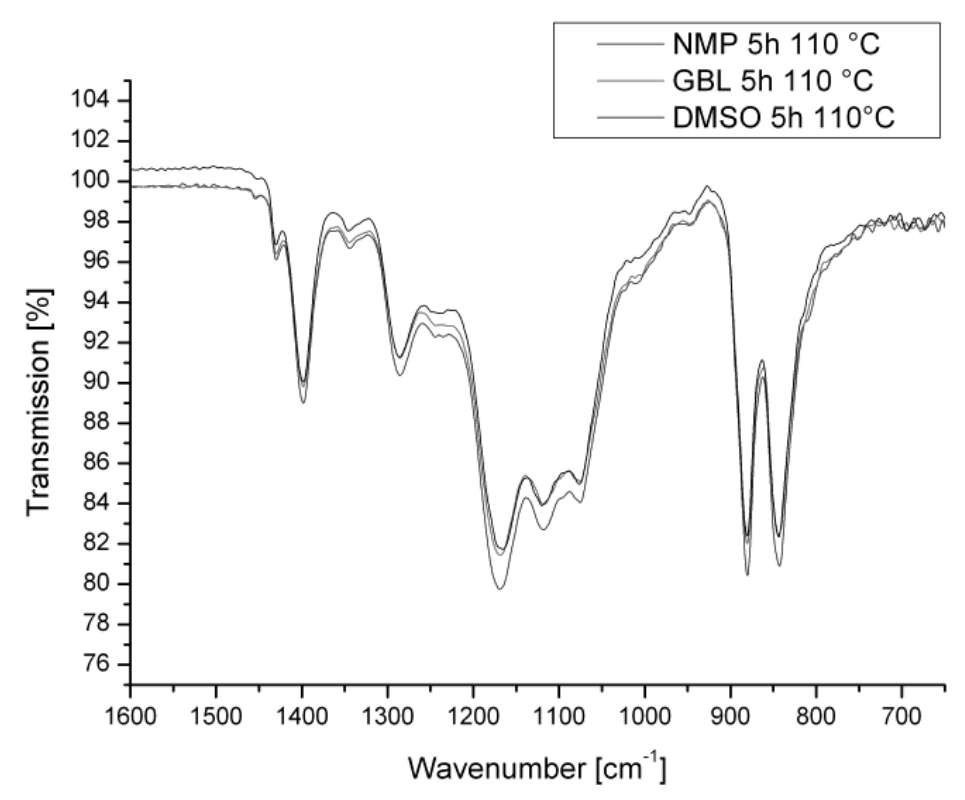

Fig.3: ATR-FTIR spectra of annealed PVDF copolymer films prepared from precursor solutions based on various solvents. Only bands of the $\beta$-phase were detected. 
The rheological behaviour of PVDF copolymer solutions in GBL was investigated. A polymer content of 18 wt.-\% was chosen and the viscosity was measured in dependence of shear rate (Fig.4).

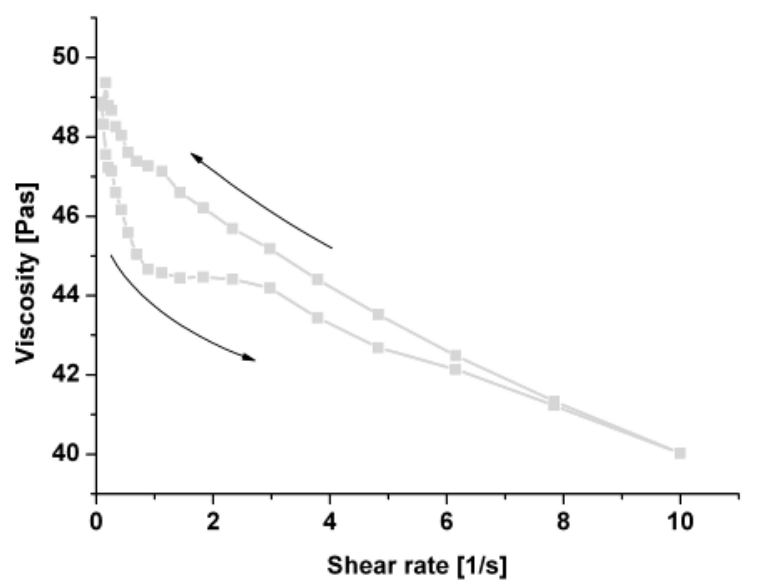

Fig.4: Rheological behaviour of a PVDF copolymer $\mathrm{y}$-butyrolactone solution (solids content 18 wt.-\%).

The viscosity of the solution lies in the range of 40 to $50 \mathrm{~Pa} \cdot \mathrm{s}$ and shows week shear thinning behaviour. But for good results in screen printing the effect has to be more pronounced. For that reason the solution was further diluted to enable low viscosity at high shear forces. As a solvent butyl acetate was chosen.

To enable high viscosity at low shear forces the diluted precursors were modified with a rheological additive. At first the applicability of fumed silica was tested. An 18 wt.-\% PVDF copolymer solution based on GBL was diluted with 60 wt.- $\%$ butyl acetate. Fumed silica was added in various amounts. Concentrations between 3 and $9 \mathrm{wt} . \%$ were tested. The rheological properties of the modified solutions were investigated using a rotational viscometer.

Fig.5 shows the plot of the viscosity for an initial low shear range (shear rate $0.1 \mathrm{~s}^{-1}$ ) followed by a high shear rate segment $\left(10 \mathrm{~s}^{-1}\right)$ and a subsequent low shear rate measurement $\left(0.1 \mathrm{~s}^{-1}\right)$. The measurement simulates the behaviour of the solution under low and high shear rates which corresponds to the printing process. The recorded values for the viscosity in the third segment show the time and level of rearrangement after applying high shear forces.

The PVDF copolymer solution modified with 3 wt.-\% fumed silica shows a strong shear thinning behaviour. The viscosity falls down to about $2 \mathrm{~Pa} \cdot \mathrm{s}$ and relaxes within about $4 \mathrm{~min}$ to $90 \%$ of the initial value.

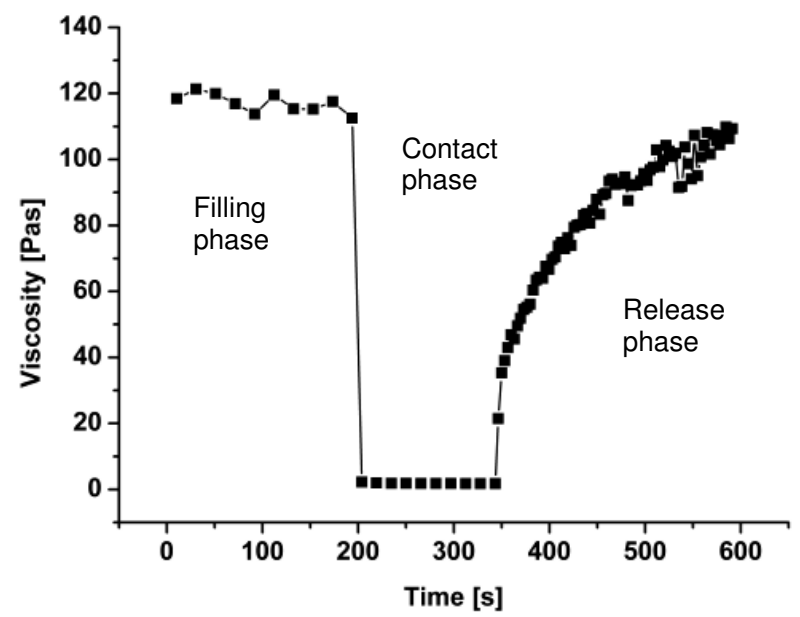

Fig. 5: Rheological behaviour of a PVDF copolymer solution diluted with 60 wt.-\% butyl acetate and modified by $3 \mathrm{wt} . \%$ fumed silica. The viscosity was measured in three segments applying shear rates of $0.1,10$ and $0.1 \mathrm{~s}^{-1}$. 
A higher amount of additive increases the low shear viscosity. After applying high shear forces the relaxation time is shorter but the initial values are not reached again (see Fig. 7).

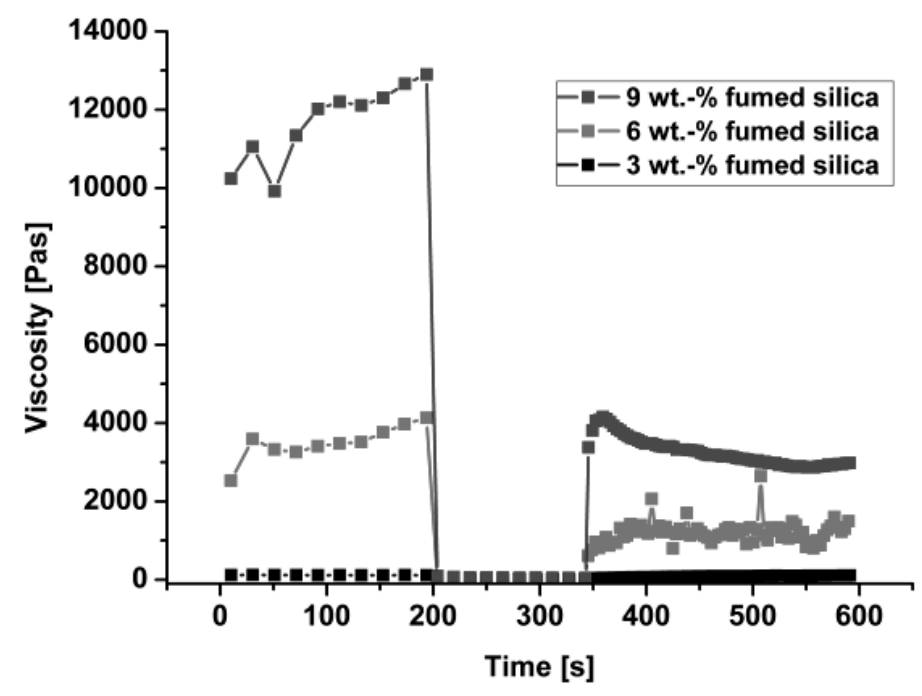

Fig. 6: Comparison of rheological behaviour of solutions modified with varied amounts of additive (fumed silica).

To conclude, the rheological properties of PVDF copolymer solutions were successfully modified by dilution and the addition of fumed silica as an additive. Shear thinning behaviour could be demonstrated. Further experiments have to clarify influences of the additive on the crystallisation behaviour and the quality of thin films prepared from the precursors.

\section{Materials for thin film transistors}

Thereafter, a gate dielectric has to be applied on the sensor materials and, subsequently, an organic semiconductor when choosing a bottom-gate architecture of the TFT (In the case of a top-gate architecture the process sequence is inverted). The thin-film dielectric should be applied by means of printing techniques such as gravure printing or ink-jet printing. However, in order to achieve organic TFTs which are able to switch the TFT at low voltages the dielectric has to be applied as thin layer. One possibility is the use of inorganic-organic hybrid polymers (ORMOCER ${ }^{\circledR} s$ ) [4] in order to realize ink-jet inks or gravure printing pastes. ORMOCER ${ }^{\Theta}$ s are synthesized by alkoxysilanes which are hydrolysed and polycondensed. The polycondensates are three-dimensional cross-linked nano-scaled oligomers which are functionalized by organic polymerisable groups. Thereby, a storage-stable resin is produced which can be cured by UV light when photo-initiators are added to the formulation before application. Due to the fact that ORMOCER ${ }^{\circledR}$ resins are liquid, there is a large scope to adjust the rheology and the wetting behavior by solvents and additives. Thereby, very thin films could already be realized $(<100 \mathrm{~nm})$ by spin-coating processes which are electrical dense and provide organic functionalities on the surface supporting the performance of the semiconductor in terms of mobility and threshold voltages (see fig 8). At present, ORMOCER ${ }^{\circledR}$ materials are adjusted to be applied as thin layer by printing processes. The ORMOCER ${ }^{\circledR}$ s excel by high dielectric strength and adjustable dielectric permittivities. Besides, in contrast to some high-performance purely organic dielectrics (such as BCB) low temperatures can be used to cure the materials. This is due to the fact that ORMOCER ${ }^{\circledR}$ are organically cross-linked by UV light resulting in a solid layer which can be post-baked at moderate temperatures (between $80^{\circ} \mathrm{C}$ and $180^{\circ} \mathrm{C}$ ). A further advantage of that hybrid polymer is in the capability to tune chemically the surface of the thin dielectric layer by the use of certain functionalized educts. This might be important, in particular, when a bottom-gate architecture is chosen. In this case, the surface of the dielectric also determines the quality of the growth of organic semiconducting molecules such as pentacene. 


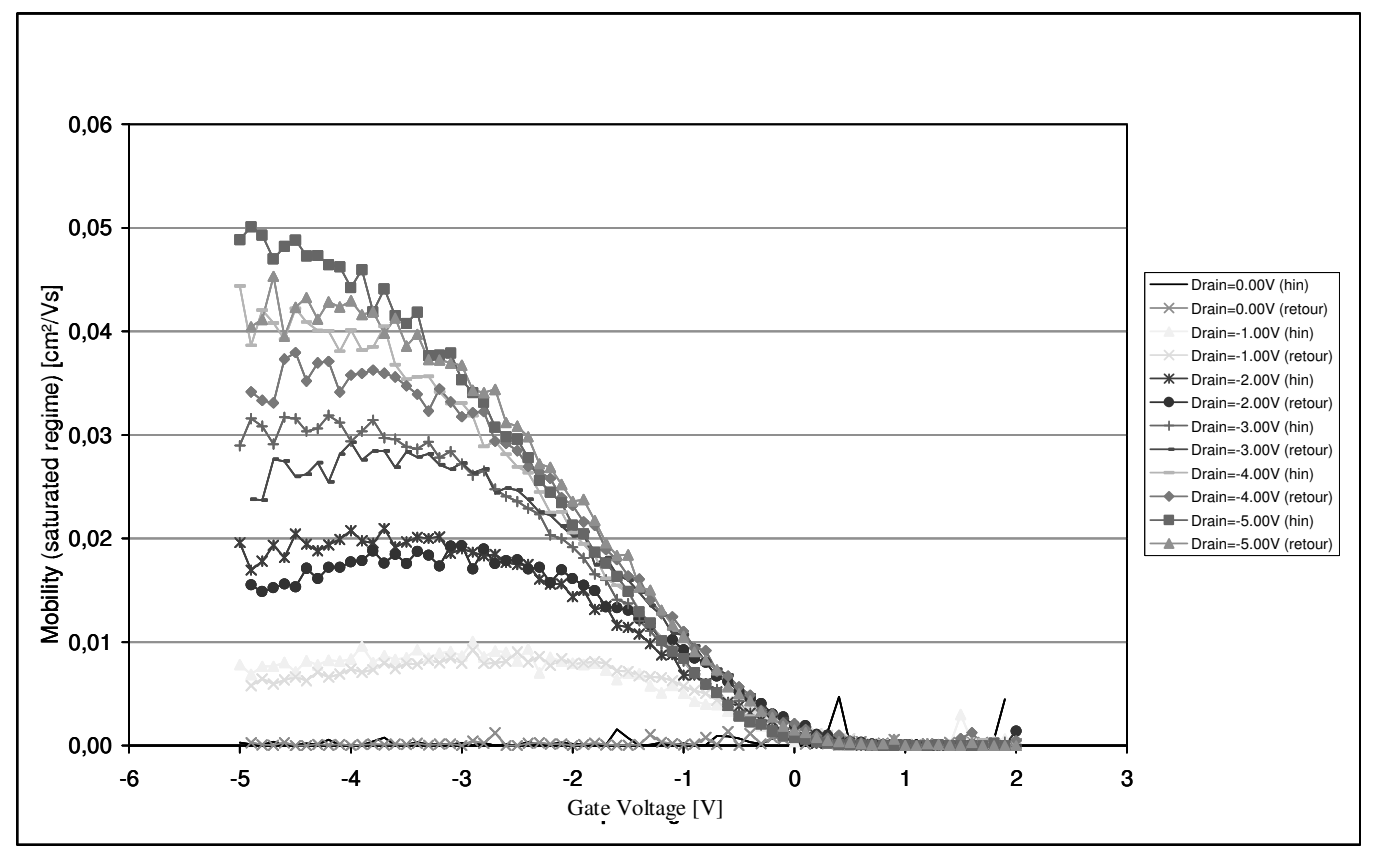

Figure 8: Input characteristics of thin film transistor manufactured by pentacene as organic semiconductor and ORMOCER $^{\circledR} \mathrm{S}$ as gate dielectric indicating that low gate voltages can be used in order to control the TFT.

Pentacene can be evaporated on the sensor element by means of in-line vacuum processes. Alternatively, P3HT based polymers can be chosen which might be applied by ink-jet technologies. Since ordinary P3HT based organic semiconductors are not very stable against humidity and oxygen, modifications of P3HT could be used in order to avoid degradation effects in terms of semiconducting properties of the material [5]. In summary, the manufacturing of cost-efficient large-area sensor devices can be achieved by a thorough development and formulation of materials towards the required properties but also to the processing techniques. The presented work will result in a sensor technology which offers a vast amount of possibilities for commercial applications but might scientifically fascinate by the underlying physics and chemistry.

These sensors are developed in an R\&D project called 3Plast which is funded by the European Union (Grant No 215036, see www.3plast-sensor.eu).

\section{Literature}

[1] M. Zirkl, A. Haase, A. Fian, H. Schön, C. Sommer, G. Jakopic, G. Leising, B. Stadlober, I. Graz, N. Gaar, R. Schwödiauer, S. Bauer-Gogonea, and S. Bauer, Adv. Mat. 19, 2241 (2007).

[2] D. Nilsson, N. Robinson, M. Berggren, and R. Forchheimer, Adv. Mat. 17, 353, (2005).

[3] Stadlober, B. ; Zirkl, M. ; Leising, G. ; Gaar, N. ; Graz, I. ; Bauer-Gogonea, S. ; Bauer, S:. IEEE, Vol.13 (5), (2006).

[4] K.-H. Haas, Adv. Eng. Mat., 2, No. 9, (2000).

[5] S. Janietz, T. Egorov-Brening, B. Gruber, Presentation on MRS Fall Meeting, (2008). 